\title{
The Antimalarial Action of Desferal Involves a Direct Access Route to Erythrocytic (Plasmodium falciparum) Parasites
}

\author{
Mark Loyevsky, Simon D. Lytton, Brenda Mester, ${ }^{*}$ Jacqueline Libman, ${ }^{*}$ Abraham Shanzer, ${ }^{*}$ and Z. loav Cabantchik \\ Department of Biological Chemistry, The Hebrew University of Jerusalem, Jerusalem, Israel 91904; \\ and ${ }^{*}$ Department of Organic Chemistry, Weizmann Institute of Science, Rehovot, Israel 76100
}

\begin{abstract}
We designed the $\boldsymbol{N}$-methylanthranilic-desferrioxamine (MADFO) as a fluorescent iron(III) chelator with improved membrane permeation properties. Upon binding of iron(III), MADFO fluorescence is quenched, thus allowing traceability of drug-iron(III) interactions. MA-DFO is well tolerated by mammalian cells in culture. Its antimalarial activity is pronounced: $\mathrm{IC}_{50}$ values on in vitro (24-h) growth of Plasmodium falciparum were $3 \pm 1 \mu \mathrm{M}$ for MA-DFO compared with $30 \pm 8$ for DFO. The onset of growth inhibition of rings or trophozoites occurs 2-4 h after exposure to $13 \mu \mathrm{M}$ MA-DFO. This effect is commensurate with MA-DFO permeation into infected cells. In a 24-h exposure to MA-DFO or DFO, trophozoites take up either compound to $\sim \mathbf{1 0} \%$ of the external concentration, rings to $5 \%$, and noninfected cells to $<1 \%$. Red cells encapsulated with millimolar concentrations of DFO or MADFO fully support parasite invasion and growth. We conclude that extracellular MA-DFO and DFO gain selective access into parasites by bypassing the host. The rate-limiting step is permeation through the parasite membrane, which MA-DFO accomplishes faster than DFO, in accordance with its higher hydrophobicity. These views are consistent with the proposed duct, which apparently provides parasitized cells with a window to the external medium. (J. Clin. Invest. 1993. 91:218-224.) Key words: erythocyte $\bullet$ permeability $\bullet$ drug delivery $\bullet$ malaria • Plasmodium falciparum • iron • antimalarial • chelators • chemotherapy • fluorescence
\end{abstract}

\section{Introduction}

The family of hydroxamate siderophores of which desferrioxamine (DFO) ${ }^{1}$ is a most prominent member has provided useful therapeutic agents for the management of pathological conditions associated with metal inbalances and versatile tools for studying iron metabolism and toxicity in mammalian cells (1).

Address correspondence to Z. Ioav Cabantchik, Department of Biological Chemistry, The Hebrew University of Jerusalem, Jerusalem, Israel 91904.

Received for publication 15 April 1992 and in revised form $14 \mathrm{Au}$ gust 1992.

1. Abbreviations used in this paper: DFO, desferrioxamine; $\mathrm{IC}_{50}$, half maximal inhibitory concentration; MA, $N$-methylanthranilate; NBD, nitrobenzyl-diazole; RBC, red blood cell; RCC, red cell container; RSF, reversed siderophores.

J. Clin. Invest.

(C) The American Society for Clinical Investigation, Inc. 0021-9738/93/01/0218/07 \$2.00

Volume 91, January 1993, 218-224
Recently it was shown that DFO congeners display effective antimalarial activity both in vitro (2-5) and in vivo (4, 6-9), but as with many other drugs, their mode of action has remained elusive (1). The information available indicates that DFO acts without affecting serum $(4,10)$ or normal red cell iron pools $(3,10)$, that it gains a restricted access to infected cells ( 11 ), and that its antimalarial action is manifested only in the advanced stages of parasite development and only after prolonged exposure of cells to drug $(3,12)$. All this evidence implies that DFO gains a slow and limited access to the mature stages of erythrocytic parasites where it exerts cytotoxic action on still unidentified parasite targets. Interestingly, unlike its cytostatic effect on various mammalian cells in culture (1315 ), the antimalarial effect of DFO in vitro might be similar to those of highly permeant reversed siderophores (RSFs) (10), which cause irreversible inhibition of parasite growth (10). The most hydrophobic members of this genre, when compared with DFO as antimalarials, displayed demonstrably faster (five-10-fold) action, 10-fold lower $\mathrm{IC}_{50}$, multistage activity, and no damage to mammalian cells in culture $(10,15)$.

In general, the antimalarial action of iron chelators, which act by scavenging intracellular iron, is dictated by the speed with which the agents reach the relevant intracellular pools and extract iron from critical sources (1). Selective accessibility to parasitized versus nonparasitized cells can be accomplished by drug design provided the permeation routes to parasites are identified and characterized $(16,17)$. In an attempt to understand the mode of action of DFO as an antimalarial agent, we have assessed in this work the pathways by which DFO accesses the erythrocytic parasites. To accomplish the present goal we tagged DFO at its $\mathrm{NH}_{2}$ terminus with the fluorescent $\mathrm{N}$-methylanthranilate (MA) and obtained MA-DFO (Figure 1). This derivatization was expected to be conservative of the ironbinding capacity and metal selectivity of DFO, while imparting both higher hydrophobic character for improved permeation and fluorescent properties for tracing of the drugs. The results of this study indicate that neither DFO nor MA-DFO permeate to any significant level into normal red cells or egress from red cell containers ( $\mathrm{RCCs}$ ) (which retain $>80 \%$ of their hemoglobin after resealing) loaded with drug by encapsulation. RCCs are invaded by parasites equally well as normal cells and demonstrably support parasite growth irrespective of whether they contain millimolar concentrations of drugs. The data presented indicate that neither DFO nor MA-DFO can approach the parasites from within the erythrocytes. Although both agents gain direct access to the erythrocytic parasite by bypassing the host cell cytosol (i.e., directly from the medium), MADFO apparently crosses the parasite plasma membrane faster than DFO and therefore it displays improved antimalarial activity. This idea is consistent with the recently proposed aqueous duct, which allows direct access of medium components to the parasite external surface (18). 


\section{Methods}

Chemicals. All biochemicals were from Sigma Chemical Co. (St. Louis, MO) or were the best available grade. All radiochemicals were from the Radiochemical Center (Amersham Corp., Arlington Heights, IL). DFO was procured from Ciba-Geigy (Basel, Switzerland). MADFO was synthesized from DFO ( $330 \mathrm{mg}, 0.503 \mathrm{mmol})$ dissolved in 1 $\mathrm{ml}$ of dimethylformamide and triethylamine $(73 \mathrm{mg}, 0.730 \mathrm{mmol}$ ) to which $N$-methylisatoic anhydride $(90 \mathrm{mg}, 0.588 \mathrm{mmol})$ was added. The resulting mixture was stirred at room temperature overnight, was concentrated in vacuo, and the residue was chromatographed through silica column (63-100; Woelm Pharma, GmbH, Eschwege, FRG) using $\mathrm{CH}_{2} \mathrm{Cl}_{2} / \mathrm{MeOH}(8: 2)$ as eluent to provide $100 \mathrm{mg}$ of pure MADFO. Melting point: $177-181^{\circ} \mathrm{C}$; UV (methanol): $341 \mathrm{~nm}\left(\epsilon_{\mathrm{M}} 3,410\right)$ and $256 \mathrm{~nm}\left(\epsilon_{\mathrm{M}} 6,480\right)$; IR ( $\left.\mathrm{KBr}\right): 1,625 / \mathrm{cm}(\mathrm{CO}-\mathrm{N})$; H-NMR (trifluoroacetic acid): $8.40(\mathrm{~m}, 1 \mathrm{H}, \mathrm{ArH}), 8.25(\mathrm{~m}, 1 \mathrm{H}, \mathrm{ArH}), 8.10$ $(\mathrm{m}, 2 \mathrm{H}, \mathrm{ArH}), \quad 4.30\left(\mathrm{~m}, 6 \mathrm{H}, \mathrm{CH}_{2} \mathrm{NOH}\right), \quad 3.95-\left(\mathrm{m}, 6 \mathrm{H}, \mathrm{CH}_{2} \mathrm{~N}\right), \quad 3.70-$ $\left(\mathrm{s}, 3 \mathrm{H}, \mathrm{NCH}_{3}\right), 3.65$ and $3.35\left(\mathrm{~m}, 8 \mathrm{H}, \mathrm{COCH}_{2}-\mathrm{CH}_{2} \mathrm{CO}\right), 3.0(\mathrm{~s}, 3 \mathrm{H}$,$\left.\mathrm{COCH}_{3}\right), 2.2\left(\mathrm{~m}, 12 \mathrm{H}, \mathrm{CH}_{2} \mathrm{CH}_{2} \mathrm{CH}_{2}\right)$.

The spectral properties of MA-DFO (excitation and emission) are given in Fig. 2, taken with a spectrometer (Fluorolog II; SPEX Industries Inc., Edison, NJ). MA-DFO forms stoichiometric 1:1 complexes with iron(III) and upon metal binding its fluorescence is quenched. The fluorescence properties can be restored by acid/EDTA treatment followed by alkalinization of the medium. Iron(III)-DFO and Iron(III)-MA-DFO complexes were obtained by addition of excess drug to iron ( III) aliquoted from methanolic $\mathrm{FeCl}_{3}$ solutions. Complexation was assessed by formation of hydroxamate-iron(III) characteristic absorption band at $430 \mathrm{~nm}$ or by fluorescence quenching and restoration.

The partition coefficients $\left(P_{\text {coeff }}\right)\left(\right.$ octanol/water, $\left.22^{\circ} \mathrm{C}\right)$ were determined as previously described $(10,15)$, giving values of $3.4 \pm 0.4$ for MA-DFO, $0.7 \pm 0.2$ for DFO, and $2.4 \pm 0.3$ for the MA-DFO-iron(III) complex.

Cultures. Cultures of Plasmodium falciparum (ITG-2F6 cloned strain from Brazil, obtained from Dr. L. H. Miller) were grown in culture flasks containing growth medium (RPMI 1640; Gibco, Laboratories, Grand Island, NY) supplemented with $25 \mathrm{mM}$ Hepes, $23 \mathrm{mM}$ sodium bicarbonate, $10 \mathrm{mM}$ glucose, and $10 \%$ heat-inactivated human plasma $\left(\mathrm{O}^{+}\right.$or $\left.\mathrm{A}^{+}\right)$and washed human erythrocytes $\left(\mathrm{A}^{+}\right)$at $2-2.5 \%$ hematocrit. The growth medium was replaced daily and the cultures were gassed with a mixture of $90 \% \mathrm{~N}_{2}, 5 \% \mathrm{CO}_{2}$, and $5 \% \mathrm{O}_{2}(19,20)$. Cells were usually harvested or subcultured when the parasitemia reached $15-20 \%$, as determined microscopically by thin blood smears stained with Giemsa. Trophozoite and early schizont stages were routinely isolated by the gelatin flotation method (21) or the Percoll-alanine method as described elsewhere $(20,22)$. Cultures of NIH 3 T3 cells, HT-29 cells, and NRK cells were grown as described elsewhere (10).

Encapsulated drugs. Parasite growth in resealed RCCs was obtained by addition of gelatin-isolated trophozoite-schizonts to RCC into which various materials were encapsulated. RCCs were prepared by placing a $1 \mathrm{ml}$ red blood cell suspension (70\% hematocrit) in a dialysis bag (Spectra/por 2; Spectrum Medical Industries, Los Angeles, CA) and dialyzing for 45 min against $500 \mathrm{ml}$ of $5 \mathrm{mM}$ sodium phosphate ( $\mathrm{pH} 7.0$ ) at room temperature under vigorous stirring. At the end of this period, the suspension was withdrawn and supplemented sequentially with the requisite amount of material to be encapsulated and a concentrated mixture of agents so as to attain final concentrations $(\mathrm{mM})$ of $5 \mathrm{Mg}$-ATP, $125 \mathrm{KCl}, 5$ glucose, and 5 sodium phosphate, $\mathrm{pH}$ 7.0. The resulting suspension was incubated for $45 \mathrm{~min}$ at $37^{\circ} \mathrm{C}$. For the estimation of encapsulation efficiency we have used markers such as ${ }^{3} \mathrm{H}$-sucrose $(1 \mu \mathrm{Ci} / \mathrm{ml}$, final $),{ }^{14} \mathrm{C}$-polyethylene glycol $(1 \mu \mathrm{Ci} / \mathrm{ml}$ final $)$, or preformed complexes of $\left[{ }^{59} \mathrm{Fe}\right] \mathrm{DFO}\left(3 \times 10^{5} \mathrm{cpm} / \mathrm{ml} \mathrm{final}\right)$ or [ $\left.{ }^{59} \mathrm{Fe}\right] \mathrm{MA}-\mathrm{DFO}\left(3 \times 10^{5} \mathrm{cpm} / \mathrm{ml}\right.$ final $)$ at a $5: 1$ ratio of drug/ $\mathrm{Fe}$ (III). Average entrapment efficiencies of DFO and of MA-DFO (given as concentration in RCCs vs. concentration in medium) were estimated after extensive washing of extracellular material. Cell contents were released by hypotonic lysis and then subjected to perchloric acid $(0.1 \mathrm{~N})$ precipitation and neutralization with $\mathrm{KOH}$ and Tris base ( $\mathrm{pH} 10.5$ ). Determination of hydroxamate content was done by spectrophotometry after complexation with iron(III) and of MA-DFO by

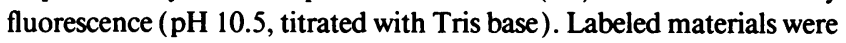
counted in a liquid $(\beta)$ scintillation counter (Beckman Instruments, Inc., Fullerton, CA). RCC hemoglobin was estimated spectrophotometrically at $540 \mathrm{~nm}$. Encapsulation efficiencies were $25-80 \%$ of the external drug concentration, with $>80 \%$ retention of cell hemoglobin. Leakage/desorption of radioactive- or fluorescent-labeled materials from RCCs was followed over a 24-h period in culture conditions (RPMI lacking indicator) both in the medium as well as in the RCCs after perchloric acid precipitation and neutralization of the supernate, as described above.

Encapsulated drugs were also assessed in terms of their capacity to inhibit parasite growth. Release of drug from RCCs was done in aseptic conditions by freezing RCCs ( $50 \%$ hematocrit in RPMI) in liquid $\mathrm{N}_{2}$ and thawing at room temperature. The suspension was centrifuged at $12,000 \mathrm{~g}$ for $8 \mathrm{~min}$ and the supernates were kept at $-20^{\circ} \mathrm{C}$ until use.

Effect on parasite growth. Upon reaching the trophozoite stage (10-20\% parasitemia), the cells were washed with growth medium, resuspended to $2 \%$ hematocrit and $1-2 \%$ parasitemia, and distributed into wells of a 24 -well microtiter plate $(0.6 \mathrm{ml}$ per well $)$. Inhibitors were added at the indicated concentrations in duplicate wells, the plate was transferred to a candle jar and incubated at $37^{\circ} \mathrm{C}$ for the indicated periods of time $(2-24 \mathrm{~h})$. After the indicated period, the supernate was removed, the cells were washed with fresh growth medium and returned to culture conditions (with or without inhibitor), so as to complete a $24-\mathrm{h}$ incubation period. Samples were withdrawn for microscopic examination of newly formed rings or trophozoites, as applicable, and $\left[{ }^{3} \mathrm{H}\right]$ hypoxanthine was added to final activity of $5 \mu \mathrm{Ci} / \mathrm{ml}$. After an additional 24-h incubation period, cells were transferred in triplicate samples to 96-well plate and harvested in a cell harvester (Dynatech Laboratories, Inc., Chantily, VA). The filters were washed with distilled water, dried for $2 \mathrm{~h}$ at $60^{\circ} \mathrm{C}$, and transferred into toluenebased scintillation fluid for counting of radioactivity. Control samples of noninfected and infected cells exposed to DMSO alone at the same final concentrations in the different systems were subjected to the same treatments as those described above. The number of equivalent experiments carried out on different blood samples was three to five.

Drug action. Stage and time dependence of drug action were assessed by exposing either ring or trophozoite cultures (1-2\% parasitemia, $2 \%$ hematocrit) to the drug for 1 to $18 \mathrm{~h}$ while following $\left[{ }^{3} \mathrm{H}\right]-$ hypoxanthine incorporation into nucleic acids. In some cases RCC-encapsulated drug or lysates derived from them were added to parasite cultures and assessed for $\left[{ }^{3} \mathrm{H}\right]$ hypoxanthine incorporation.

$\mathrm{The} \mathrm{IC}_{50}$ values of drugs on parasite growth were calculated from the profiles of parasite growth using the Dixon method (15) as published elsewhere (10).

Permeation properties of free ligands and iron complexes in normal and infected red cells. Ingress of DFO and of MA-DFO into normal and infected cells ( $10 \%$ hematocrit, $>80 \%$ parasitemia) was measured either with free drug (MA-DFO) or after complexation of DFO or MADFO (at $40 \mu \mathrm{M}$ ) with $4 \mu \mathrm{M}$ unlabeled iron(III) $\mathrm{Cl}_{3}$ and ${ }^{59} \mathrm{Fe}$ citrate (5 $\left.\times 10^{5} \mathrm{cpm} / \mathrm{ml}\right)$ in RPMI. After various times of incubation at $37^{\circ} \mathrm{C}$ (0-24 h), cells were thoroughly washed with isotonic $\mathrm{NaCl}$ containing $10 \mathrm{mM}$ EDTA, pH 7.4, and lysed with distilled water. ${ }^{59} \mathrm{Fe}$ was counted in scintillation fluid (Beckman Instruments Inc.) and fluorescent MADFO was determined after perchloric acid precipitation in the presence of EDTA followed by neutralization with $\mathrm{KOH}$ and alkalinization with Tris-Base, as described above. The number of cells was obtained from the hemoglobin absorption at $540 \mathrm{~nm}(20)$. Egress experiments on RCCs were carried out after encapsulation of normal cells with drugs ( 4 $\mathrm{mM}$ DFO and $1.3 \mathrm{mM}$ MA-DFO) and after the efflux at $37^{\circ} \mathrm{C}$ into RPMI lacking indicator ( $2 \%$ hematocrit). Total egressed material and extracellular hemoglobin were determined at different times.

\section{Results}

It is known that $\mathrm{NH}_{2}$-terminal derivatization of DFO with nitrobenzyl-diazole (NBD) does not reduce the iron(III)-bind- 
ing capacity of the drug (15). With the aim of obtaining a fluorescent and generally nontoxic DFO derivative (the NBD group conferred DFO with toxic properties to some cells in culture; our unpublished observations) we opted for the conjugation of the $\mathrm{N}$-methylanthranilic acid group, resulting in MADFO (Fig. 1). MA conferred DFO with fluorescent properties, relatively higher hydrophobicity (i.e., partition coefficient), and traceability, which was manifested as iron(III)-mediated quenching of fluorescence upon formation of the MA-DFOiron(III) complex (Fig. 2). Binding of iron(III) was essential for quenching of fluorescence since iron(III) did not quench the fluorescence of anthranilic acid conjugated to amino-dextran (not shown). Moreover, unlike NBD-DFO, MA-DFO at $50 \mu \mathrm{M}$ was demonstrably not toxic to a variety of mammalian cells in culture (not shown).

Antimalarial activity in intact cells. The MA group conferred DFO with three unique properties in terms of arrest of Plasmodium falciparum parasite growth in in vitro conditions: lower $\mathrm{IC}_{50}$ values, faster speed of action, and inhibition at both ring and trophozoite stages (Figs. 3 and 4). The speed of action of MA-DFO and DFO as antimalarial agents was examined with synchronized cultures of rings and trophozoites while following $\left[{ }^{3} \mathrm{H}\right]$ hypoxanthine incorporation into nucleic acids. The cultures consisted of midterm rings, with virtually $<1 \%$ trophozoites. Trophozoites appeared in the control cultures of rings $\sim 10-12 \mathrm{~h}$ after initiation of the experiment. The cultures of trophozoites consisted primarily of young trophozoites. As shown, the rate of incorporation in the presence of MA-DFO diverged within 4 to $6 \mathrm{~h}$ of exposure of rings and 2 to $4 \mathrm{~h}$ of exposure of trophozoites. In contrast, DFO acted on rings with less efficiency and at relatively slower speeds than MA-DFO. The $\mathrm{IC}_{50}$ values for $24 \mathrm{~h}$ of exposure of trophozoite to drug (Fig. 3) were $3 \pm 1 \mu \mathrm{M}$ for MA-DFO and $30 \pm 8 \mu \mathrm{M}$ for DFO with slightly higher values for rings.

MA-DFO and DFO were also tested for possible inhibitory effects on nucleic acid and protein synthesis in various mammalian cell lines. Similar to studies carried out with RSFs (10), no significant effects were observed by exposing cells to a 50 $\mu \mathrm{M}$ concentration of either drug for a 24 -h period (not shown).

Permeation properties. To assess whether the faster speed of action of MA-DFO relative to DFO reflected its higher octanol/water partition coefficient ( 3.4 compared with 0.7 ) and therefore its access to cell compartments, we studied the permeation properties of MA-DFO and DFO into noninfected and infected cells. We assessed those properties in terms of ingress of drug ( $40 \mu \mathrm{M})$ supplemented with traces of the respective

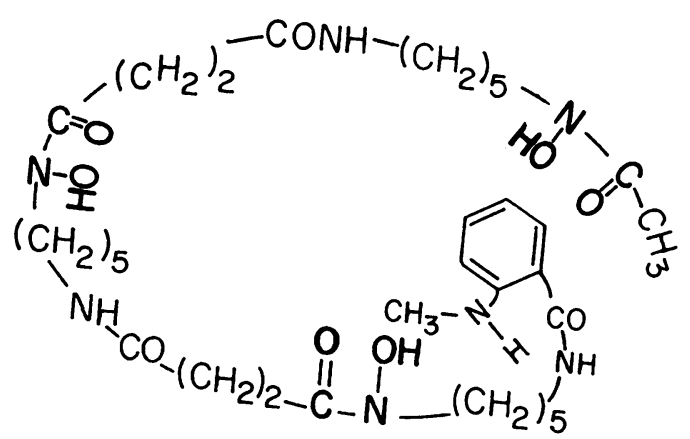

Figure 1. Schematic structure of MA-DFO. The $N$-methylanthranilic acid (MA) is coupled via an amide bond to the $\mathrm{NH}_{2}$-terminal end of DFO.
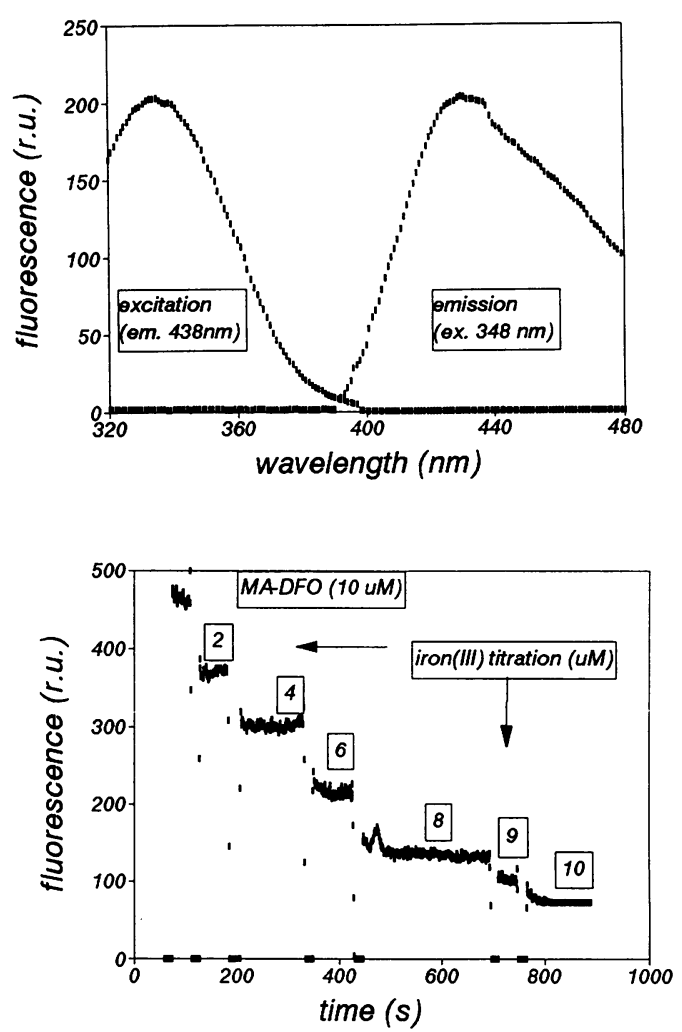

Figure 2. Fluorescence properties of MA-DFO. (Top) Excitation (left) and emission (right) spectra of a 10- $\mu \mathrm{M}$ MA solution in saline Hepes, $10 \mathrm{mM}, \mathrm{pH}$ 7.4. The nanomolar value given is for the respective wavelength of either emission (em.) or excitation (ex.) used to obtain the spectrum (noncorrected). (Bottom) Fluorimetric titration of a 10- $\mu \mathrm{M}$ MA-DFO solution with $\mathrm{FeCl}_{3}$ added from a concentrated methanolic solution (final concentrations in boxes).

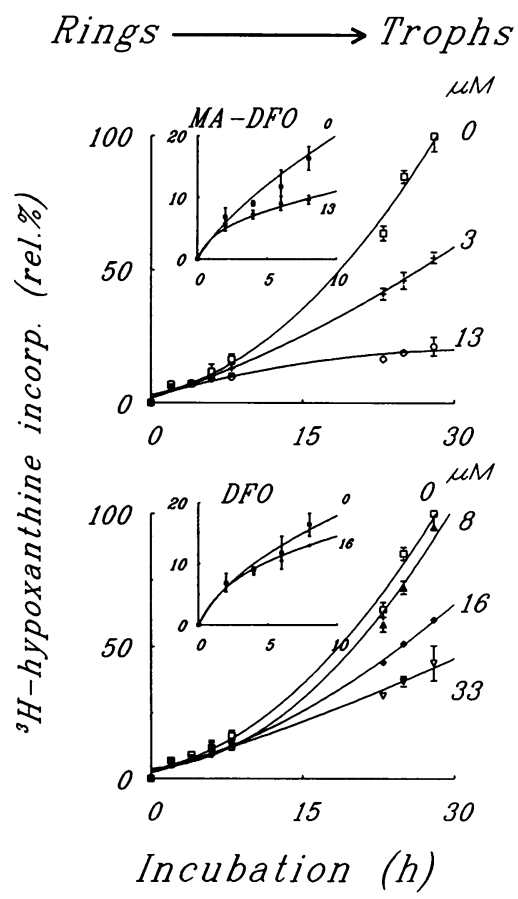

Figure 3. Continuous monitoring of DFO and MA-DFO effects on the growth of $P$. falciparum rings. The incorporation of $\left[{ }^{3} \mathrm{H}\right]-$ hypoxanthine into nucleic acids was followed in the presence of the indicated concentrations of MA-DFO (top) or DFO (bottom). All cell cultures were $2 \%$ hematocrit and 4\% rings. ${ }^{3} \mathrm{H}$ Incorporation in control samples after $24 \mathrm{~h}$ was $1.1 \pm 0.1 \times 10^{4}$ $\mathrm{dpm} /$ sample $(n=6)$. The apparent $\mathrm{IC}_{50}$ values for 0 to $8 \mathrm{~h}$ exposure were $12 \pm 3 \mu \mathrm{M}$ (MADFO) and $\gg 30 \mu \mathrm{M}$ (DFO), and for 8 to 24 $h$ exposure were $3 \pm 1$ $\mu \mathrm{M}$ (MA-DFO) and $30 \pm 8 \mu \mathrm{M}$ (DFO). The insets show data for the first $10 \mathrm{~h}$ of uptake in control and comparable concentrations of drugs used (13-16 $\mu \mathrm{M}$, as indicated). 


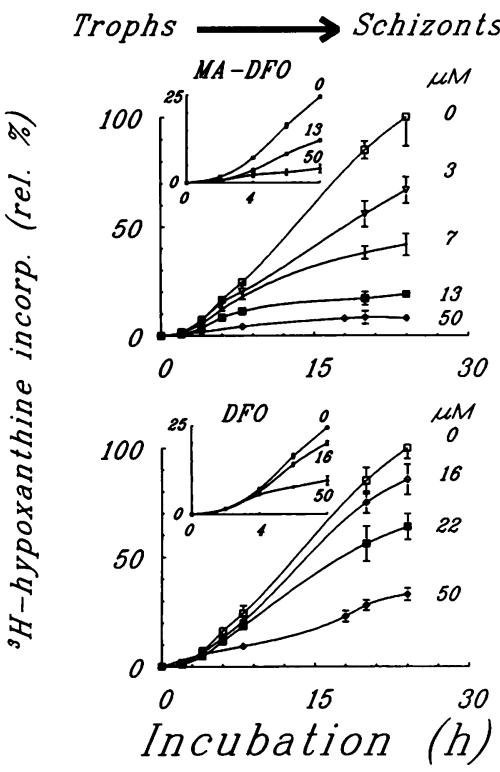

DFO) and $\gg 30 \mu \mathrm{M}$ (DFO) and for 8-24 h exposure were 4+2 $\mu \mathrm{M}$ (MA-DFO) and $33 \pm 9 \mu \mathrm{M}$ (DFO). $\mathrm{IC}_{50}$ values were calculated by Dixon plots. The insets show data for the first $10 \mathrm{~h}$ of uptake in control and comparable concentrations of drugs used (13-16 and $50 \mu \mathrm{M}$ agents, as indicated).

${ }^{59}$ iron(III) complexes as well as in terms of free MA-DFO. To the extent that the partition coefficients of the free and complexed MA-DFO are very similar ( $3.4 \pm 0.4$ and $2.4 \pm 0.3$ ), it can also be assumed that uptake of labeled complexes provides a measure for that of free chelator. Uptake studies carried out either with radioactively labeled complexes (Fig. 5 ) or free fluorescent chelator (MA-DFO) (not shown) indicated that noninfected cells were virtually impermeant to these agents over a 24-h period. A small and insignificant amount of label was apparently adsorbed on the cell surface. However, in infected cells (rings or trophozoites), time-dependent uptake was detected, giving values 3-10-fold faster for MA-DFO than for DFO. As shown in Fig. 5, the concentration of drug attained within the infected cells after $7 \mathrm{~h}$ represents only $5-15 \%$ of that in the medium. This calculation assumed no adsorption of drug to cells and the entire infected cell volume accessible to drug, thus representing the smallest possible concentration of drug attained in the parasitized cell. We assume that adsorption was dismal, since it could not be removed by washing with medium containing $10 \mathrm{mM}$ EDTA.

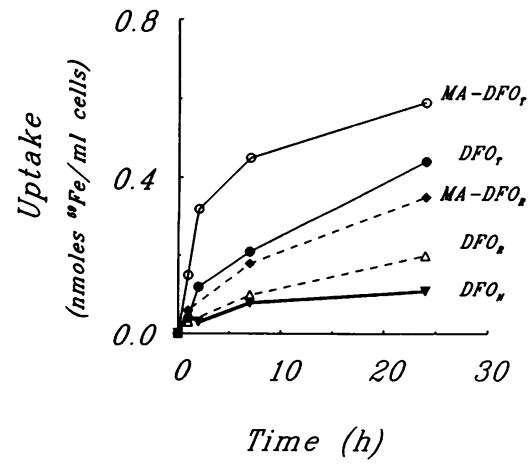

Figure 5. Ingress of ${ }^{59} \mathrm{Fe}$ complexes of DFO and MA-DFO into infected and noninfected cells. Uptake of preformed iron complexes (4- $\mu \mathrm{M}$ complexes formed with 10 -fold excess of either DFO or MA-DFO) into noninfected cells $(N)$, early trophozoites $(T)$ (>95\% parasitemia) or rings $(R)(>90 \%$

parasitemia ) was carried out at $37^{\circ} \mathrm{C}$ for the indicated periods of time as detailed in Methods.
The complementary egress experiment was carried out on RCCs because the impermeability of noninfected cells rendered them not amenable to preloading with drug. The efficiency of drug encapsulation was $20-80 \%$ of the external concentration. The RCCs also adsorbed or entrapped in their membranes a saturating amount of MA-DFO, probably during the resealing/reannealing process (Fig. 6). The level of adsorption was almost invariant with drug concentration attained inside the RCCs: $10 \%$ for MA-DFO (intracellular, $1.3 \mathrm{mM}$ ) and $<3 \%$ for DFO (intracellular, $4 \mathrm{mM}$ ). The adsorbed MA-DFO completely desorbed or leaked out from the RCCs in $<2 \mathrm{~h}$ at $37^{\circ} \mathrm{C}$, after which no release of drug was observed over a $24-\mathrm{h}$ period (hemolysis was dismal and not significantly different from that obtained with intact cells, not shown). DFO, on the other hand, showed no detectable desorption or leak over the same period of time.

Lack of antimalarial activity in DFO and MA-DFO encapsulated RCCs. RCCs are defined as hypotonically lysed/isotonically reannealed cells that retain $>80 \%$ of their hemoglobin. Drugs were encapsulated in RCCs with 25 to $80 \%$ efficiency measured chemically, radiochemically, or by bioassaying RCC lysates after extensive washing of the resealed/ reannealed cells. The RCCs support parasite invasion and growth just as well as intact cells, whether or not they are encapsulated with DFO (2-4 mM) or with MA-DFO (1.3 mM) (Fig. 7). A direct proof for invasion into RCCs containing DFO or MA-DFO (millimolar levels) was obtained by labeling the drug containing RCCs with traces of drug- ${ }^{59} \mathrm{Fe}$ complexes (at micromolar levels). $24 \mathrm{~h}$ after addition of trophozoites to RCCs, the newly formed rings were segregated from noninfected cells by the Percoll-sorbitol method and the ${ }^{59} \mathrm{Fe} / \mathrm{he}-$ moglobin ratio was compared with drug-free RCCs (Fig. 7, bottom). As seen, very similar values were obtained for all types of RCCs, indicating similar invasion into cells irrespective of whether they contained the drug. The inhibitory effect of material released from RCCs was precisely the same as that observed with free drug added to cells. This proves not only that the drugs were indeed encapsulated but also that they were (at least initially) available for biological action should they become accessible to the relevant parasite compartments.

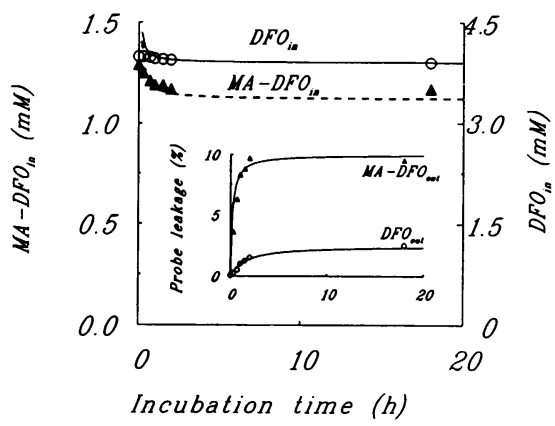

Figure 6. Egress of MA-DFO and DFO from RCCs. Egress of DFO or MA-DFO with time was followed from RCCs loaded with either 4 $\mathrm{mM}$ DFO or $1.3 \mathrm{mM}$ MA-DFO by the method of encapsulation; hematocrit was $2.5 \%$ in RPMI lacking indicator at $37^{\circ} \mathrm{C}$. Agents appearing outside were measured spectroscopically as detailed in Methods; data in inset (leakage of probe) are given in terms of percent of material (DFO or MA-DFO) originally present in RCCs after encapsulation and washing of extracellular agents. The main graph shows data of drug retained in RCCs (mM) as a function of time. 


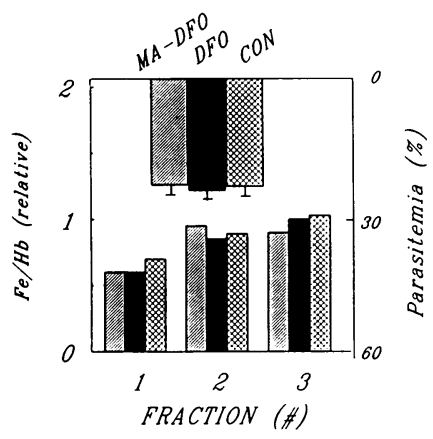

determined for the three systems (top bar were separated by Percoll gradients into three fractions and the ratio of ${ }^{59} \mathrm{Fe} / \mathrm{Hb}$ was determined after lysis (bottom bar graph): fraction 3 noninfected cells, fraction 2 early rings, and fraction 1 late rings; control RCCs (crossed hatched bars), DFO encapsulated ( filled bars), and MA-DFO encapsulated (empty bars).

To directly demonstrate that parasites not only invaded but also developed inside drug-containing RCCs, we followed their growth in terms of nucleic acid synthesis (Fig. 8). At millimolar concentrations, encapsulated drugs did not interfere with the intraerythrocytic development of parasites whereas drug added extracellularly was inhibitory even at 100 -fold lower concentrations. Any inhibitory effect found with encapsulated drug, particularly MA-DFO, could be fully explained by the presence of drug in the outer medium, as exogenously supplemented iron(III) abrogated those effects. That drug derived most probably from RCCs by leakage or desorption, as shown previously in Fig. 6. Moreover, if RCCs loaded with drugs were washed and used as targets for parasite invasion and growth after drug leak ceased $\left(2 \mathrm{~h}\right.$ incubation at $37^{\circ} \mathrm{C}$; Fig. 6$)$, no inhibitory effect of encapsulated MA-DFO was obtained (not shown). Finally, a direct proof that encapsulated MA-DFO and DFO were not neutralized by red cell cytosol components was obtained by testing the RCC lysates on parasite growth. As shown in Fig. 8, the inhibitory capacity of these drugs was largely retained in the drug-containing RCCs whereas shamloaded RCC lysates had virtually no effect at equivalent dilutions (1:100 of original lysates). The fact that inhibition was completely eliminated by addition of exogenous iron(III) to RCC lysates indicated that the active component of the lysates had iron(III)-binding capacity.

\section{Discussion}

The study of the mode of action of antimalarial agents is important for the design of novel therapeutic tools, which are in urgent demand (23). This truism also applies to iron chelators, which have already scored success in the management of various diseases (1), including malaria (9). To gain knowledge on the mode of action of iron chelators as antimalarial agents and to determine the basis for their specific effects on infected cells it is necessary to delineate the routes by which they permeate into cells and to identify the critical iron pools. Our limited understanding reflects in part the lack of reliable probes for detection of the drugs, and in the case of malaria, for tracing their fate in a highly compartmentalized cell such as the $P$. falciparum-infected cell. We have recently undertaken the initiative to design fluorescent derivatives of iron chelators by
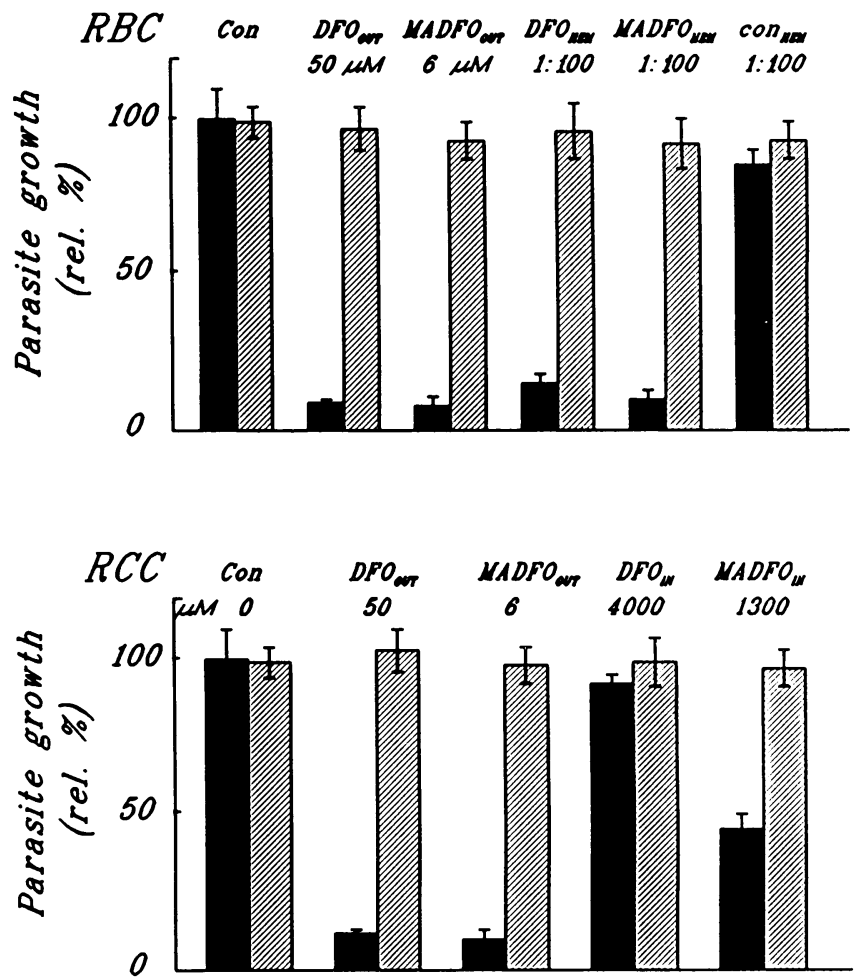

Figure 8. Effect of intracellular versus extracellular MA-DFO and DFO. (Top) The effects of extracellular DFO DFO $_{\text {OUT }}(6 \mu \mathrm{M})$ were assessed in cultures of parasites grown in red blood cells $(\mathrm{RBC})$. Gelatin-enriched trophozoites were added to RBCs, incubated for $24 \mathrm{~h}$, and subsequently supplemented with $\left[{ }^{3} \mathrm{H}\right]-$ hypoxanthine as described in Methods. After an 18-h incubation period, cells were harvested and incorporation of label into nucleic acids was determined. Data are given as percent incorporation relative to control (parasite growth rel. \%). The effect of hemolysates (HEM) ( 1:100 final dilution) of RCCs encapsulated with none (Con), $4 \mathrm{mM}$ DFO, or $1.3 \mathrm{mM}$ MA-DFO (all final concentrations in RCCs) were also assessed on trophozoite cultures grown on RBC. Bars represent systems supplemented with (hatched) or without (filled) $100 \mu \mathrm{M}$ $\mathrm{FeCl}_{3}$, respectively. (Bottom) Gelatin-enriched trophozoites were also added to RCCs containing none (Con), 4,000 $\mu \mathrm{M} \mathrm{DFO}_{\mathrm{IN}}$ or 1,300 $\mu \mathrm{M}$ MA-DFO ${ }_{\mathrm{IN}}$ ( final internal concentrations), as described above for RBC. After $24 \mathrm{~h}$ incubation, $\left[{ }^{3} \mathrm{H}\right]$ hypoxanthine was added and the suspension was incubated for an additional 18-h period. Extracellular concentrations of $\mathrm{DFO}_{\text {OUT }}$ and MA-DFO on control RCCs as indicated above for RBCs. Data are given as for RBCs but relatively to control RCCs. Values of parasite growth in control RBCs and control RCCs were essentially the same $( \pm 10 \%)$.

means conservative of their iron-binding properties (15). The classical agent DFO was previously derivatized with the NBD group to yield NBD-DFO (15), and in this study we attached the MA group to yield MA-DFO; in both cases the fluorescent tags were conjugated to the $\mathrm{NH}_{2}$ terminus of DFO. The parent DFO was chosen as the backbone not only because of its remarkable antimalarial activity in vitro $(2-5)$ and in vivo $(4$, $6-9)$, as well as with human subjects (9), but also because it offered the possibility of chemical and biological improvements by judicious chemical modifications. DFO was specifically modified with the methylanthranilic group at its $\mathrm{NH}_{2}$-terminal group, so as to confer to it a higher octanol/water partition coefficient and therefore higher permeation through 
membranes without compromising the iron-binding properties of the hydroxamate core. The resulting MA-DFO, although less fluorescent than the NBD congener NBD-DFO $(15,24)$, lacked cytotoxic activity on mammalian cells in culture (NIH3T3 and NRK fibroblasts, HT-29 human colon carcinoma cells, and Hep-2 human hepatoma cells; not shown).

The novel MA-DFO showed improved antimalarial performance when compared with DFO as manifested by its 10 -fold lower $\mathrm{IC}_{50}$, faster speed of action, and activity on both early and mature stages of parasite growth. We attributed these improvements to the higher partition coefficient conferred by the MA group, which in turn increased its permeation into infected cells. Moreover, the structural changes in DFO did not significantly alter the permselectivity of infected and noninfected cells for the derivatized DFO. Although the differential performance of MA-DFO and DFO was manifested in the speed of action of these drugs on trophozoites, the same could not be unequivocally said about the effects on rings shown in Fig. 3. The possibility that a minor undetectable fraction of early trophozoites or late rings might have been present in the predominantly ring culture could not be dismissed. Due to the relatively higher metabolic capacity of trophozoites compared with rings, incorporation of label into nucleic acid might have been dominated by the contaminant population. However, the fact that in rings the onset of drug inhibitory activity was $\geq 2-4 \mathrm{~h}$ later than in trophozoites served as an indication that the observed effects were obtained on genuine rings rather than on contaminating trophozoites.

One of the salient features of DFO and MA-DFO uptake into infected cells is that even after hours of incubation with drug only $15 \%$ of the external concentration is attained within the infected cell (assuming the entire cell volume as drug available space). Similar results were previously reported in a study involving ${ }^{14} \mathrm{C}-\mathrm{DFO}$ (11), which demonstrated a threefold higher permeation into infected compared with noninfected cells. The apparent restricted permeation of DFO and MADFO into infected cells raises the question of which compartment(s) are accessed by DFO and MA-DFO and which are the relevant membranes they need to cross to reach those compartments. The accepted view depicts the parasite enclosed in the red cell and bound by a parasitophorus vacuole membrane (Fig. 9). To gain access into parasite, drugs would need to cross several membranes and diffuse through various aqueous compartments. Previous biophysical analysis led to a complex picture with multiple and distinct pathways of entry into the parasitized erythrocyte and the enclosed parasite $(16,17)$. A recent study proposed a novel pathway of access of substances into the parasite via an aqueous duct leading to parasite outer membrane (18). The validity of that pathway has been questioned (25) but firmly defended (26). Clearly exogenous agents can gain access to the parasitized cell as a whole by alternative or parallel routes that might lead to different intracellular compartments or even to the same compartments by a combination of parallel and serial routes. From within the host cell cytosol, the agents can gain access to the parasite compartments only by sequential crossing of the parasitophorous vacuole membrane (or duct membrane), diffusion in the intermembranous space, and crossing the parasite plasma membrane (Fig. 9). Small and lipophilic molecules could accomplish that by simple diffusion mechanisms, but less lipophilic molecules or macromolecules might need to be trans-

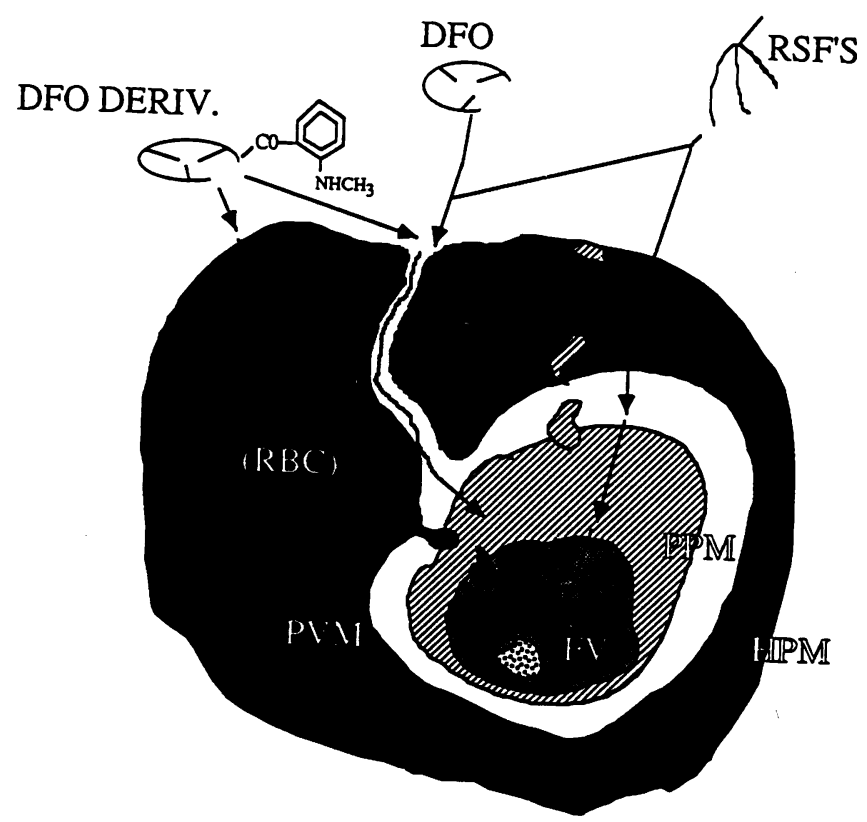

Figure 9. Model of access routes of DFO and MA-DFO into infected cells. The model depicts the host cell with its plasma membrane $(H P M)$, the parasite with its plasma membrane $(P P M)$ and its food vacuole $(F V)$, and the duct (18) that invaginates from the red cell membrane and surrounds the parasite forming the parasitophorous vacuole membrane $(P V M)$. Compounds such as DFO and MA-DFO gain access to the parasite preferentially through the duct and the PPM. RSFs, which are permeant or reversed siderophores, can gain intracellular access both via the HPM and the PPM.

ported by endocytotic mechanisms, as demonstrated for hemoglobin (27). From outside the parasitized cell, macromolecules such as dextrans and ferritin were recently implied to be taken up into parasites by endocytosis $(18,28)$, although neither the chemical fate of such molecules nor the pharmacological relevance of that uptake mechanism have been assessed. Another study indicated that a $70-\mathrm{kD}$ dextran-bound DFO encapsulated in RCCs failed to inhibit parasite growth at $100-\mu \mathrm{M}$ concentrations (but was inhibitory at higher concentrations). This agent displayed a $\mathrm{IC}_{50}$ of $\sim 180 \mu \mathrm{M}$ when applied extracellularly (29), but since its chemical stability was not assessed it is not clear whether the extracellular effect was exerted by the macromolecular DFO or by a breakdown product. In our study we have clearly demonstrated that DFO and MA-DFO, both of which were inhibitory to parasites at micromolar concentrations when applied extracellularly, demonstrably failed to inhibit parasite invasion and growth when placed in the erythrocyte cytosol even at millimolar concentrations. The results are consistent with the notion that both DFO and MA-DFO enter the parasite through the proposed duct and permeate into the parasite. We suggest that crossing of the parasite plasma membrane is diffusive in nature, in accordance with the higher permeation properties of MA-DFO compared with the more hydrophilic DFO. Entry via this pathway might not only explain the selective admittance of DFO and its derivatives into parasites but also the apparently limited permeation of these compounds to parasitized erythrocytes, since only the duct and/or the parasite show accessibility to those agents. NBD-DFO and the lipophilic RSFs, because of their higher partition coeffi- 
cients, could access the parasite either through the duct or partially via the host cell.

The validity of the conclusions drawn from studies based on RCCs loaded with DFO and MA-DFO (encapsulated drugs) as targets for parasite invasion and growth depends also on firm evidence that the drugs encapsulated in RCCs were retained after $24 \mathrm{~h}$ in culture conditions as chemically detectable entities and more importantly as biologically active agents. Both types of information are provided in this work. Lysates of RCCs loaded with drugs display inhibitory activities that correspond to $\sim 70 \%$ of the equivalent concentrations of free drugs. This partial reduction in biological activity might be associated with drug interaction with hemoglobin, leading to hydroxamate-induced formation of methemoglobin (Loyevsky, M., and Z. I. Cabantchik, unpublished observations), as shown in previous studies with DFO (29). Thus at present we can not eliminate the possibility that the high hemoglobin content of RCCs can in situ abrogate the biological activity of the drugs via reversible chemical interactions (i.e., reversible upon dilution). In terms of the mode of entry of the agents into infected cells, any DFO-like drugs that enter the host cell cytosol would become virtually ineffective for parasite arrest as a result of one of the following possibilities: inaccessibility to parasites, chemical interactions with red cell components, or chemical modifications. The results of the present study are consistent with the proposed parasite membranous duct as the most feasible route for access of DFO-like chelators to parasites. Further understanding of the permeation properties of DFO derivatives into parasites and their intracellular fate in parasites and in host cells can pave the road for novel antimalarial drug design or improvement of the existing repertoire, as shown in this work.

As the antimalarial activity of all the DFO derivatives are abrogated by complexation with iron(III), it is implied that extraction of iron(III) from a parasite target mediates the cytotoxic activity. Erythrocytic parasites might not be able to efficiently restore the chelated iron and are therefore assumed to be irreversibly damaged by iron chelators of the hydroxamate families (30).

\section{Acknowledgments}

The work was supported in part by the E. D. Bergman Memorial Fund (Jerusalem) (Dr. Cabantchik), World Health Organization DIF (Dr. Cabantchik), the Israel Ministry of Science and Technology (Drs. Cabantchik and Libman), and Makor Chemical Co., Jerusalem (Dr. Shanzer). Dr. Shanzer is a holder of a Sigfrid and Irma Ullman professorial chair.

\section{References}

1. Hershko, C., and D. J. Wheatherall. 1988. Iron chelating therapy. CRC Crit. Rev. Clin. Lab. Sci. 26:303-345.

2. Raventos-Suarez, C., S. Pollack, and R. L. Nagel. 1982. Plasmodium falciparum: inhibition of in vitro growth by desferrioxamine. Am. J. Trop. Med. Hyg. 31:919-922.

3. Whitehead, S., and T. E. A. Peto. 1990. Stage-dependent effect of deferoxamine on growth of Plasmodium falciparum in vitro. Blood. 76:1250-1255.
4. Hershko, C., and T. E. A. Peto. 1988. Deferoxamine inhibition of malaria is independent of host iron status. J. Exp. Med. 168:375-387.

5. Peto, T. E. A., and J. L. Thompson. 1986. A reappraisal of the effects of iron and desferrioxamine on the growth of Plasmodium falciparum in vitro: the unimportance of serum iron. Br. J. Haematol. 63:273-280.

6. Yinnon, A. M., E. N. Ihenacho, R. W. Grady, D. T. Spira, and C. Hershko. 1989. Antimalarial effect of HBED and other phenolic and catecholic iron chelators. Blood. 74:2166-2171.

7. Pollack, S., R. N. Rossan, D. E. Davidson, and A. Escajadillo. 1987. Desferrioxamine suppresses Plasmodium falciparum in Aotus monkeys. Proc. Soc. Exp. Biol. Med. 184:162-164.

8. Fritsch, G., J. Treumer, D. T. Spira, and A. Jung. 1985. Plasmodium vinckei: suppression of mouse infections with desferrioxamine B. Exp. Parasitol. 60:171-174.

9. Traore, O., P. Carnevalle, L. Kaptue-Noche, J. M'Bede, M. Desfontaine, J. Elion, D. Labie, and R. L. Nagel. 1991. Preliminary report on the use of desferrioxamine in the treatment of Plasmodium falciparum malaria. Am. J. Hematol. 37:206-208.

10. Shanzer, A., J. Libman, S. D. Lytton, H. Glickstein, and Z. I. Cabantchik. 1991. Reversed siderophores act as antimalarial agents. Proc. Natl. Acad. Sci. USA. 88:6585-6589.

11. Fritsch, G., and A. Jung. $1986 .{ }^{14} \mathrm{C}$-Desferrioxamine B: uptake into erythrocytes infected with Plasmodium falciparum. Z. Parasitenkd. 72:709-713.

12. Carter, T., M. T. Bayne, V. R. Gordeuk, G. M. Brittenham, and A. Masamichi. 1991. Stage-specific ultrastructural effects of desferrioxamine on Plasmodium falciparum in vitro. Am. J. Trop. Med. Hyg. 45:593-601.

13. Lederman, H. M., A. Cohen, J. W. W. Lee, M. H. Freedman, and E. W. Gelfand. 1984. Deferoxamine: a reversible S-phase inhibitor of human lymphocyte proliferation. Blood. 64:748-753.

14. Peto, T. E. A., and C. Hershko. 1989. Iron and infection. In Bailliere's Clinical Haematology. 2.2:435-321.

15. Lytton, S. D., Z. I. Cabantchik, J. Libman, and A. Shanzer. 1991. Reversed siderophores act as antimalarial agents: II. A fluorescent derivative of desferal, NBD-desferal, selectively scavenges iron (III) from parasitized red cells. Mol. Pharmacol. 40:584-590.

16. Cabantchik, Z. I. 1989. Altered membrane transport of malaria-infected erythrocytes: a possible pharmacologic target. Blood. 74:1464-1471.

17. Cabantchik, Z. I. 1990. Properties of permeation pathways induced in the human red cell membrane by malaria parasites. Blood Cells (NY). 16:421-432.

18. Pouvelle, B., R. Spiegel, L. Hsiao, R. J. Howard, R. L. Morris, A. P. Thomas, and T. F. Taraschi. 1991. Direct access to serum macromolecules by intraerythrocytic malaria parasites. Nature (Lond.). 353:73-75.

19. Trager, W., and J. B. Jensen. 1976. Continuous culture of human malaria parasites. Science (Wash. DC). 193:673-675.

20. Kutner, S., W. V. Breuer, H. Ginsburg, J. B. Aley, and Z. I. Cabantchik. 1985. Characterization of permeation pathways in plasma membrane of human erythrocytes infected with early stages of Plasmodium falciparum: association with parasite development. J. Cell. Physiol. 125:521-527.

21. Jensen, J. B. 1978. Concentration from continuous culture of erythrocytes infected with trophozoites and schizonts of Plasmodium falciparum. Am. J. Trop. Med. Hyg. 27:1274-1276.

22. Rivadeneira, E. M., M. Wasserman, and C. Espinal. 1983. Separation and concentration of schizonts of Plasmodium falciparum by Percoll gradients. $J$. Protozool. 30(2):367-370.

23. Wernsdorfer, W. H. The development and spread of drug-resistant malaria. 1991. Parasitol. Today. 7:297-303.

24. Lytton, S. D., B. Mester, J. Libman, A. Shanzer, and Z. I. Cabantchik. 1992. Monitoring of iron(III) removal from biological sources using a novel fluorescent siderophore. Anal. Biochem. 205:326-333.

25. Sherman, I. W., and R. Zidovetzky. 1992. A parasitophorus duct in Plasmodium-infected red blood cells. Parasitol. Today. 8:2-3.

26. Taraschi, T. F., B. Pouvelle, and R. J. Howard. 1992. A parasitophorus duct in Plasmodium-infected red blood cells: a reply. Parasitol. Today. 8:17.

27. Yayon, A., R. Timberg, S. Freedman, and H. Ginsburg. 1984. Effects of chloroquine on the feeding mechanism of the intraerythrocytic human malarial parasite Plasmodium falciparum. J. Protozool. 31(3):367-372.

28. Burns, E. R., and S. Pollack. 1988. P. falciparum infected erythrocytes are capable of endocytosis. In Vitro Cell. Dev. Biol. 24:481-486.

29. Scott, M. D., A. Ranz, F. A. Kuyper, B. Lubin, and S. R. Meshnick. 1990. Parasite uptake of desferroxamine: a prerequisite for antimalarial activity. Br. $J$. Haematol. 75:598-602.

30. Lytton, S. D., B. Mester, I. Dayan, H. Glickstein, J. Libman, A. Shanzer, and Z. I. Cabantchik. 1993. Mode of action of iron (III) chelators as antimalarials. I. Membrane permeation properties and cytotoxic activity. Blood. In press. 